\title{
Elaboration et validation d'un modèle simple de mélange des eaux en estuaire. Application au Bou Regreg (Côte atlantique marocaine)
}

\section{J. Massio 1}

Mots-clés : estuaire, débits résiduels, modèle de mélange, dispersion, Maroc.

A partir de données morphologiques et de mesures de salinité, un modèle numérique de mélange des eaux dans l'estuaire du Bou Regreg a été élaboré. L'estuaire a été divisé en 23 tronçons d'un kilomètre de long pour lesquels le modèle permet de calculer l'évolution dans le temps des salinités moyennes à mi-marée. Au cours de la période étudiée, les échanges entre les 23 tronçons paraissent largement dominés par la dispersion. Le modèle reproduit correctement l'évolution de la salinité après une crue artificielle dûe à un lâcher de barrage mais également dans d'autres situations.

Elaboration and validation of a simple mixing water model for an estuary. Application to the Bou Regreg (Moroccan atlantic coast)

Keywords : estuary, salinity, residual discharges, operating models, dispersion, Morocco.

Using morphological data and salinity measurements, a numerical model describing the mixing of water along the Bou Regreg estuary was developed. The estuary has been divided into 23 sections, each $1 \mathrm{~km}$ in length.

The model can be used to calculate the temporal changes of the average salinity for each section at midtide. During the study period the exchanges between the 23 sections were largely governed by dispersion. The model reproduces correctly the salinity variations after a major release of fresh water from an upstream reservoir and also in other situations.

\section{Introduction}

Dans tout estuaire soumis à la marée, il existe des mouvements d'eau " majeurs » assez facilement mesurables : débit fluvial, entrée et sortie d'eau au cours de chaque marée.

Il existe, en même temps, des débits que l'on ne peut mesurer qu'indirectement : ce sont des débits résiduels (ce qui resterait si on pouvait faire abstraction du va-et-vient de la marée). L'évaluation de ces débits « mineurs " est d'un très grand intérêt, en particulier lorsqu'il y a introduction et dispersion dans l'estuaire de substances polluantes dissoutes.

Dans le cas où le débit fluvial est très faible par rapport aux courants de marée, c'est la dispersion qui devient responsable de l'essentiel des échanges au sein de l'estuaire. Elle est généralement exprimée

1. Laboratoire d'Hydrobiologie, URA 695 C.N.R.S., Université Paul Sabatier, 118, route de Narbonne, F-31062 Toulouse Cedex, France. en terme de coefficients de dispersion mais dans la pratique, on peut également la représenter par des flux s'établissant entre des tronçons d'estuaire supposés homogènes.

L'étude des estuaires marocains est en cours d'actualisation mais il n'existe encore pour l'estuaire du Bou Regreg qu'un modèle permettant de calculer les hauteurs d'eau en fonction de la marée (Mayif 1987) et une étude préliminaire concernant le déplacement des masses d'eau (Dahbi 1988).

L'élaboration de modèles de fonctionnement faciles à mettre en œuvre, applicables aux estuaires situés dans les zones de climat sub-humide à semiaride très peuplées, est devenue une nécessité. En effet, dans ces régions, on peut constater de plus en plus souvent une dégradation accélérée de la qualité des eaux aussi bien littorales qu'estuariennes. Des modèles simples de prédétermination peuvent apporter, entre autres, une aide considérable pour la réalisation d'aménagements touchant au traitement et au rejet des eaux usées. 


\section{Milieu physique}

Le Bou Regreg se jette dans l'océan Atlantique entre les villes de Rabat et de Salé ( $34^{\circ}$ Nord, $6^{\circ} 50^{\prime}$ Ouest) dans une région peuplée par près de 1 million d'habitants.

Le climat local est de type méditerranéen subhumide. Les précipitations (566 mm), enregistrées à Salé pour l'année 1986 au cours de laquelle ont été effectuées la plupart des mesures servant à la modélisation, ont été voisines de la moyenne calculée par Gillet (1987) pour les vingt dernières années $(585 \mathrm{~mm})$.
L'estuaire mesure 24 kilomètres de long entre l'embouchure et le pied du barrage Sidi Mohammed Ben Abdallah, mis en service en 1973, qui marque la limite de la marée dynamique (Fig. 1).

Dans sa partie amont, l'estuaire entaille sur 8 kilomètres les derniers rebords de la Messeta Côtière et se trouve parfois dominé par des falaises hautes de près de 100 mètres. Les 15 kilomètres de la zone aval se situent dans une plaine alluviale légèrement resserrée au voisinage de l'embouchure et orientée SENW.

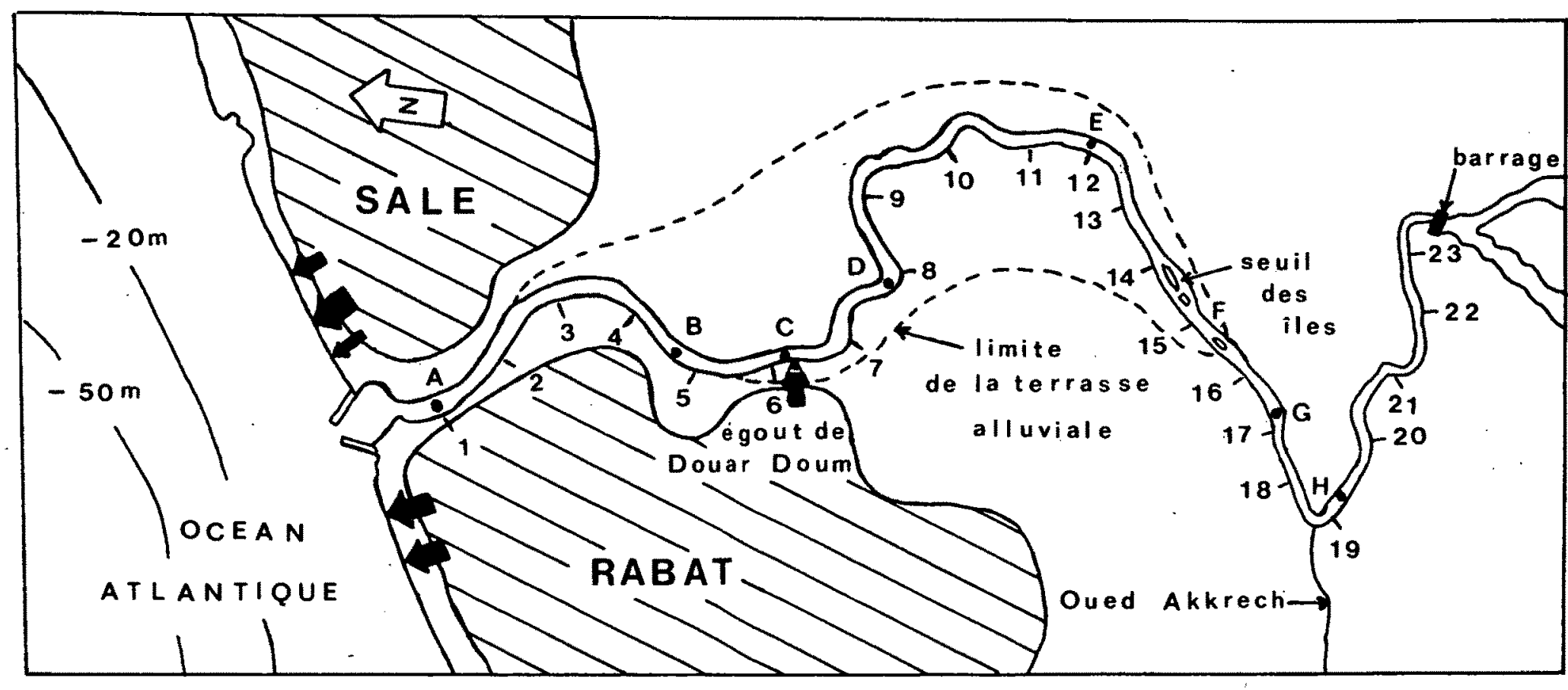

A

Localisation des principaux émissaires d'égouts

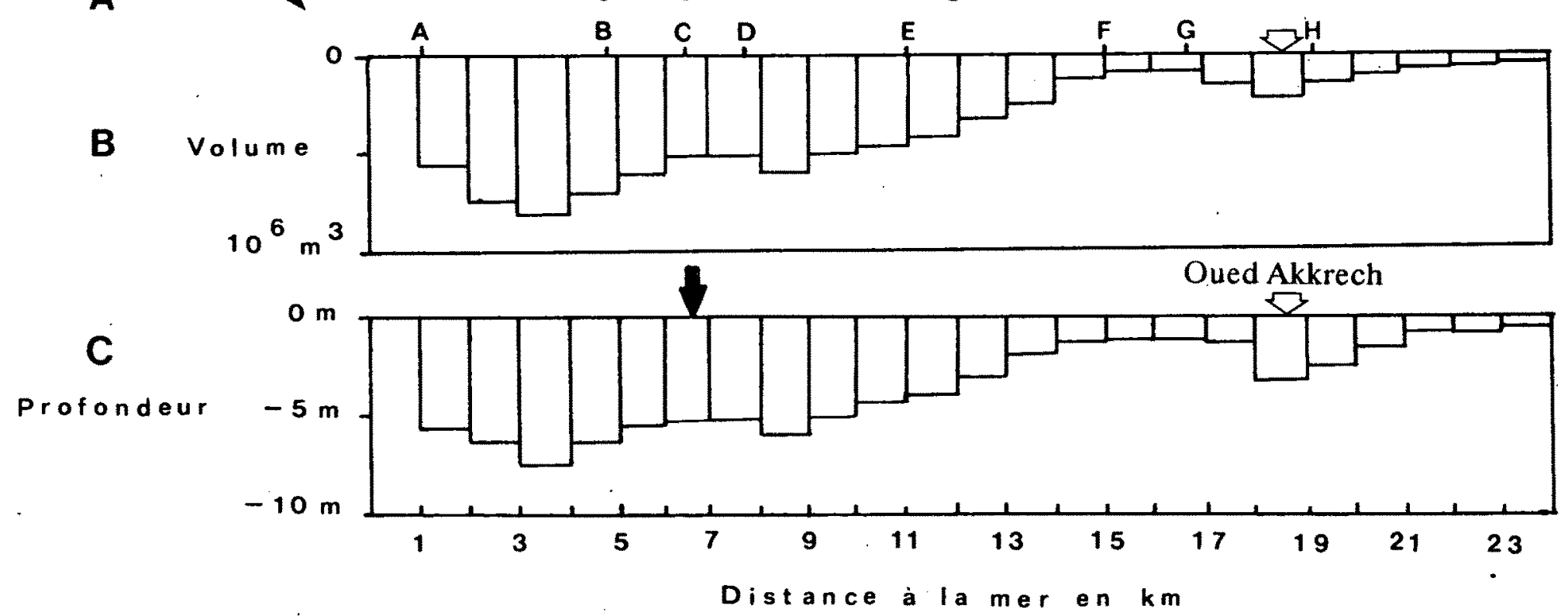

Fig. 1. A : Carte de l'estuaire du Bou Regreg et localisation des sites de prélèvements notés de A à H. Les limites des 23 tronçons pris en compte sont indiquées par la distance en $\mathrm{km}$ par rapport à la mer. B : Volume de chaque tronçon de $1 \mathrm{~km}$ à mi-marée. $C:$ Profondeur moyenne de chaque tronçon de $1 \mathrm{~km}$ à mi marée.

Fig. 1. A : Map of the Bou Regreg estuary and location of the sampling stations named from A to $\mathrm{H}$. The limits of the 23 sections taken into account are indicated by the distance from the sea in $\mathrm{km}$. B : Volume of each $1 \mathrm{~km}$ section at midtide. C : Average depth of each $1 \mathrm{~km}$ section at midtide. 
La marée est semi-diurne de période $12 \mathrm{~h} 15 \mathrm{mn}$. Son amplitude maximum est de 3,3 $\mathrm{m}$ et son amplitude minimum de $0,5 \mathrm{~m}$.

Pour la marée moyenne (amplitude $=2 \mathrm{~m}$ ) le débit moyen à l'embouchure $\left(\simeq 300 \mathrm{~m}^{3} . \mathrm{s}^{-1}\right)$ représente environ 500 fois le débit d'eau douce qui parvient dans l'estuaire en dehors des périodes d'apports exceptionnels. En effet, le débit d'eau prélevé dans la retenue du barrage pour l'alimentation des villes de Rabat et de Casablanca $\left(9 \mathrm{~m}^{3} . \mathrm{s}^{-1}\right.$ en 1983) représente près de $50 \%$ du débit moyen annuel de l'Oued Bou Regreg et dépasse largement le débit moyen annuel des années les plus sèches (4 à 5 $\mathrm{m}^{3} . \mathrm{s}^{-1}$. De ce fait, même au cours des années humides, l'estuaire n'est la plupart du temps alimenté en eau douce que par les fuites du barrage, par un très petit cours d'eau (l'Oued Akkrech) se jetant directement dans la partie amont de l'estuaire et par les égoûts de certains quartiers de Rabat et de Salé.

En raison de la réduction des apports en eau douce, l'estuaire est presque constamment du type verticalement homogène selon la classification de Pritchard (1955). On peut toutefois constater quelques différences de salinité entre la surface et le fond mais elles restent très limitées (Texier et al. 1986, Mayif 1987). La salinité totale est voisine de celle de l'eau de mer (29 à $33 \mathrm{~g} .1^{-1}$ à l'embouchure) dans les 6 premiers kilomètres de la zone aval pui elle diminue jusqu'au pied du barrage où il ne reste pratiquement que de l'eau douce (Tableau 1, Fig. 2).

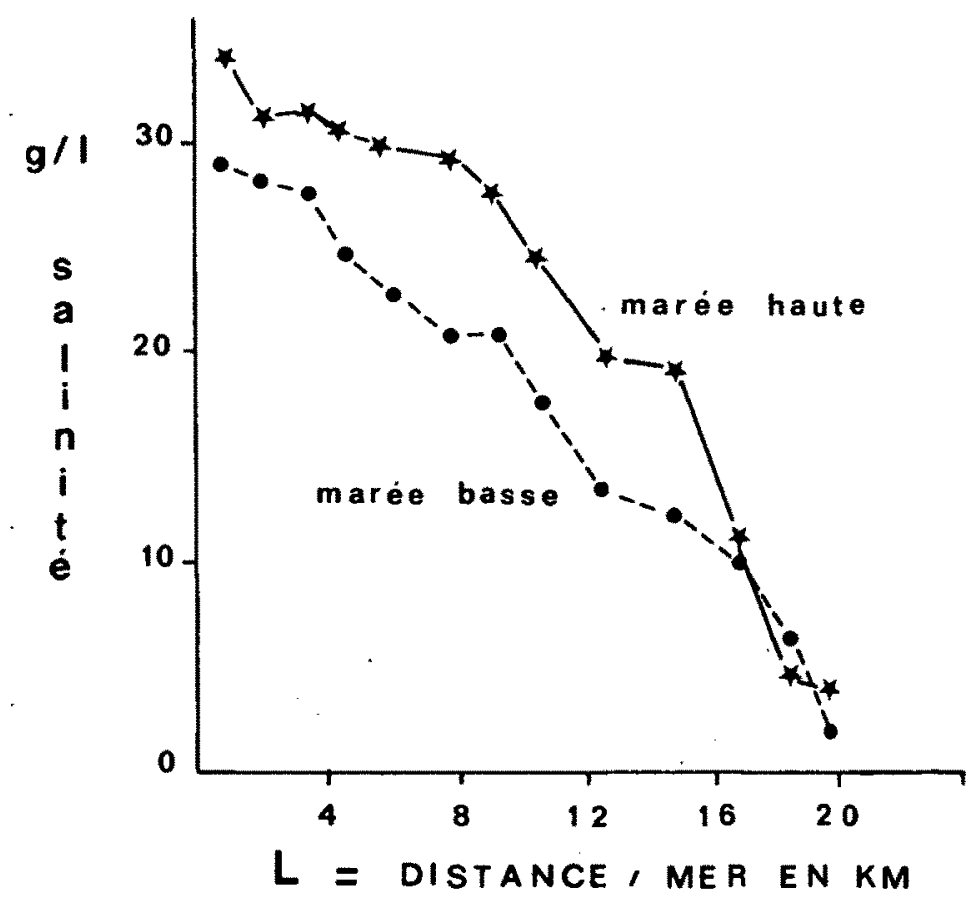

Fig. 2. Répartition de la salinité à marée haute et à marée basse dans l'estuaire du Bou Regreg lors d'une marée d'amplitude $1,7 \mathrm{~m}$.

Fig. 2. Salinity distribution at high tide and low tide in the Bou Regreg estuary during a tide with an amplitude of $1,7 \mathrm{~m}$.

Tableau 1. Eléments utilisés pour l'élaboration et la validation du modèle de mélange longitudinal.

Table 1. Elements used for the elaboration and the validation or the longitudinal mixing model.

\begin{tabular}{|c|c|c|c|c|c|c|c|}
\hline $\begin{array}{l}\text { Portion } \\
\mathrm{N}^{\circ} \\
\end{array}$ & $\begin{array}{c}\text { Volume } \\
\text { Portion } \\
\mathrm{m}^{3} \\
\end{array}$ & $\begin{array}{c}\text { Salinité } \\
\text { à } t+9 j \\
g /\end{array}$ & $\begin{array}{c}\text { Salinité } \\
\text { à } t+49 \mathrm{j} \\
\mathrm{g} / 1\end{array}$ & $\begin{array}{c}\text { Apports } \\
\text { eau } \\
\text { douce } \\
\mathrm{m}^{3} / \mathrm{h} \\
\end{array}$ & $\begin{array}{c}\text { Débit des } \\
\text { échanges } \\
\mathrm{D} 1+\mathrm{D} 2 \\
\mathrm{~m}^{3} / \mathrm{s}\end{array}$ & $\begin{array}{c}\text { Salinité } \\
\text { à } t+49 \mathrm{j} \\
\text { Mod } 1 \\
\mathrm{~g} / 1\end{array}$ & $\begin{array}{c}\text { Salinité } \\
\text { à } t+49 \mathrm{j} \\
\text { Mod } 2 \\
\mathrm{~g} / 1\end{array}$ \\
\hline
\end{tabular}


A mi-marée, pour un volume moyen de $8,3 * 10^{6}$ $\mathrm{m}^{3}$, la largeur moyenne de l'estuaire naturel est de $115 \mathrm{~m}$ et sa profondeur moyenne de $4 \mathrm{~m}$.

Le volume d'eau marine entrant dans l'estuaire au cours de la marée moyenne (amplitude $=2 \mathrm{~m}$ ) est de $7^{*} 10^{6} \mathrm{~m}^{3}$ ce qui correspond alors à plus de $50 \%$ du volume d'eau présent dans l'estuaire à marée haute.

L'estuaire comprend deux parties très distinctes séparées par une zone de hauts fonds : le seuil des îles (km 15). Dans la partie amont, les courbes de hauteur de marée sont extrêmement déformées et la durée du jusant se trouve considérablement allongée par rapport à celle du flot. C'est ainsi qu'au niveau du seuil des îles, le flot ne dure en moyenne que 4,25 heures alors que le jusant se maintient 8 heures et que l'on passe à des durées de 3 heures pour le flot et de 9,25 heures pour le jusant au confluant de l'Oued Akkrech (km 18) (Elkaim 1972, Texier et al. 1986).

\section{Modèles mathématiques de circulation et de mélange des eaux dans les estuaires}

Les premiers modèles mathématiques de circulation et de mélange des eaux dans les estuaires ont été proposés au début des années 50 . En raison de la très grande diversité des types d'estuaires et de la variabilité des phénomènes régissant leur fonctionnement (hauteur de marée, débit fluvial...), il est rapidement apparu qu'il serait très difficile de transposer les premiers modèles généraux, comme celui de Arons \& Stommel (1951), à des estuaires très différents les uns des autres.

Les modèles semi-empiriques se sont par contre, dès le départ, révélés de bons outils de travail, aussi bien pour les estuaires verticalement homogènes (Ketchum 1951) que pour les estuaires à deux couches avec entraînement (Bowden 1967). Les premiers modèles utilisaient un découpage de l'estuaire en tronçons de longuer importante correspondant à l'intrusion marine (Ketchem 1951, Preddy 1954). Certains modèles plus tardifs comme celui de Dorestein (1960) (in Bowden 1967) ont utilisé un découpage en tronçons beaucoup plus courts.

Depuis cette époque, la modélisation des estuaires a beaucoup progressé en raison des avancées sur la connaissance de ce type de milieu, du développement des outils numériques et de l'augmentation des capacités de calcul. Les approches de la modélisation se sont également diversifiées pour s'adapter au mieux aux conditions locales et aux objectifs poursuivis (Ulanowicz 1976).

Les modèles de type analytique ont cherché à prendre en compte de façon de plus en plus complète tous les phénomènes qui influencent le fonctionnement des estuaires. C'est le cas par exemple de la circulation transversale pour Fischer (1972), du renforcement de la stratification entre l'amont et l'aval d'un estuaire pour Elliott (1976), de la possibilité d'alternance de phases de mélange et de stratification au cours d'une même marée pour Simpson et al (1990) ou encore des caractéristiques morphologiques majeures propres à chaque estuaire pour Geyer \& Signel (1990).

Les modèles semi-empiriques comme celui de Ketchum (1951) ont également été perfectionnés (Dyer \& Taylor 1973) et restent fréquemment utilisés (Brown \& Arellano 1980, Bradley et al 1990). Ces modèles donnent des résultats satisfaisants à condition d'être soigneusement étalonnés.

\section{Choix et description du type de modèle utilisé}

\subsection{Comportement de l'estuaire au cours de la période considérée}

De façon à mieux caractériser les estuaires, de nombreux indices, calculés à partir de grandeurs physiques assez facilement mesurables, ont été proposés.

Un des indices les plus simples est celui de Schultz \& Simmons (1957) in Hansen \& Rattray (1966). Il $s$ 'agit du rapport $R$ entre la somme des apports d'eau douce au cours d'une marée (ici $12,4 \mathrm{~h}$ ) et le prisme de marée défini comme le volume d'eau salée entrant pendant le flot. En général, lorsque le rapport $\mathrm{R}$ est inférieur à 0,1 , l'estuaire peut être considéré comme verticalement homogène. Toutefois, Bradley et al (1990) ont montré que la valeur limite de $\mathrm{R}$ doit être ramenée à 0,05 si la morphologie de l'estuaire favorise la stratification. Si l'on adopte cette limite, le calcul montre que l'estuaire du Bou Regreg peut devenir partiellement mélangé lorsque le débit d'eau douce est supérieur à $10 \mathrm{~m}^{3} / \mathrm{s}$ pour une marée moyenne (amplitude $=2 \mathrm{~m}$ ) et à $3,5 \mathrm{~m}^{3} / \mathrm{s}$ pour une marée de mortes eaux (amplitude 0,9 m). En dehors 
des périodes de lâcher du barrage, ces débits d'apports d'eau douce ne sont atteints que très exceptionnellement.

Pritchard (1967) propose une limite inférieure de l'indice R de Schultz \& Simmons beaucoup plus basse, de l'ordre de 0,001 , ce qui correspond à un débit d'eau douce de $0,2 \mathrm{~m}^{3} / \mathrm{s}$ pour l'estuaire du Bou Regreg. Dans ces conditions, l'estuaire serait encore verticalement homogène pendant toute la saison sèche.

Il est également possible de calculer le nombre d'estuaire E de Harleman \& Abraham (1966) (in Meyer-Branski, 1981).

$\mathrm{E}=\mathrm{Pt} \times \mathrm{Uo}^{2} / \mathrm{T} \times \mathrm{g} \times$ ho $\times$ Qf

avec $: P_{t}=$ prisme de marée

$U_{o}=$ vitesse maximum du flot à l'entrée de l'estuaire

$\mathrm{T}=$ période de la marée

$\mathrm{g}=$ accélération de la pesanteur

ho $=$ profondeur moyenne à l'entrée de l'estuaire

$\mathrm{Qf}=$ débit d'eau douce

Ces deux auteurs ont indiqué qu'un estuaire est bien mélangé lorsque $E>0,1$. Cette valeur correspond, pour l'estuaire du Bou Regreg, à un apport d'eau douce de $17 \mathrm{~m}^{3 / \mathrm{s}}$ au cours d'une marée moyenne (amplitude $2 \mathrm{~m}$ ).

Fischer (1972) a montré que la stratification dépend essentiellement du nombre de Richardson d'estuaire $\mathrm{RiE}=\mathrm{g} \times \Delta \mathrm{d} \times \mathrm{Qf} / \mathrm{b} \times$ do $\times \mathrm{Uo}^{3}$ avec : $\Delta \mathrm{d}=$ contraste de densité entre l'eau de mer et l'eau douce

do = densité de l'eau de mer

$\mathbf{b}=$ largeur moyenne du chenal à l'entrée de l'estuaire

Pour l'estuaire du Bou Regreg, avec une marée moyenne de $2 \mathrm{~m}$ d'amplitude et un apport d'eau douce de $0,45 \mathrm{~m}^{3} / \mathrm{s}, \mathrm{RiE}=0,0055$. Ce chiffre correspond d'après Meyer-Branski (1981) reprenant les travaux de Fischer, à la situation dans laquelle le zoefficient de dispersion moyen ne se trouve plus influencé par la stratification.

L'estuaire du Bou Regreg peut donc être consiJéré comme verticalement homogène durant la plus zrande partie de l'année même si, comme le soulizne Pritchard (1967), l'homogénéité parfaite n'existe jas dans la réalité. Les courants résiduels, même s'ils iont très faibles, restent classiquement orientés vers 'amont près du fond et vers l'aval près de la surface
(Elkaïm 1972, Mayif 1987) de sorte que les courbes d'isosalinité moyenne se trouvent légèrement incurvées vers l'amont au voisinage du fond. Ces faibles différences de salinité entre le fond et la surface ne semble avoir, la plupart du temps, que très peu d'influence dans la limitation des échanges verticaux. Dans un estuaire comme celui du Bou Regreg les phénomènes de dispersion prennent ainsi largement le pas sur les phénomènes de transfert.

\subsection{Choix du type de modèle utilisé}

En dehors des périodes d'apports d'eau douce exceptionnellement élevés, un modèle semiempirique d'évolution de la salinité dans un estuaire homogène ou très faiblement stratifié peut donc être utilisé pour le Bou Regreg. Dans la pratique, lorsque l'on peut se satisfaire de modèles relativement simples et faciles à mettre en œuvre, les modèles filaires ou unidimensionnels peuvent suffire pour représenter des estuaires ou des portions d'estuaire non stratifiés (Meyer-Branski 1981, Bradley et al. 1990, Salomon et al. 1991).

Le pas de temps entre deux séries de résultats exploitables, doit être également adapté au type d'application envisagé. En ce qui concerne les études de sédimentologie ou d'écotoxicologie comme celles qui ont été réalisées dans l'estuaire du Bou Regreg par Mahif (1987), Cheggour $(1988,1990)$ et Dahbi (1988), il n'est pas nécessaire, dans un premier temps, de rechercher une précision à l'échelle d'une marée ni d'étudier l'effet à court terme des variations du coefficient de marée. Il paraît suffisant de calculer des valeurs moyennes de salinité à mi-marée sachant que les résultats obtenus coïncident précisément avec la réalité physique avec une périodicité de 15 jours. Ce pas de temps correspond à un intervalle entre deux marées moyennes incluant une période de vives eaux et une période de mortes eaux.

Toutefois, pour obtenir une intégration correcte de tous les phénomènes, un pas de temps de 1 heure doit être utilisé pour les calculs.

Un modèle morphologique, représentant l'estuaire à mi-marée, a été élaboré par Dahbi (1988) à partir de données anciennes (Elkaim 1972) complétées par des travaux récents (Gillet, 1986 ; Mayif 1986 ; Cheggour 1987). Ce modèle est composé de 23 tronçons d'un kilomètre de long constituant l'estuaire naturel (Tableau 1, Fig. 1). Le $24^{\mathrm{e}}$ tronçon aval 


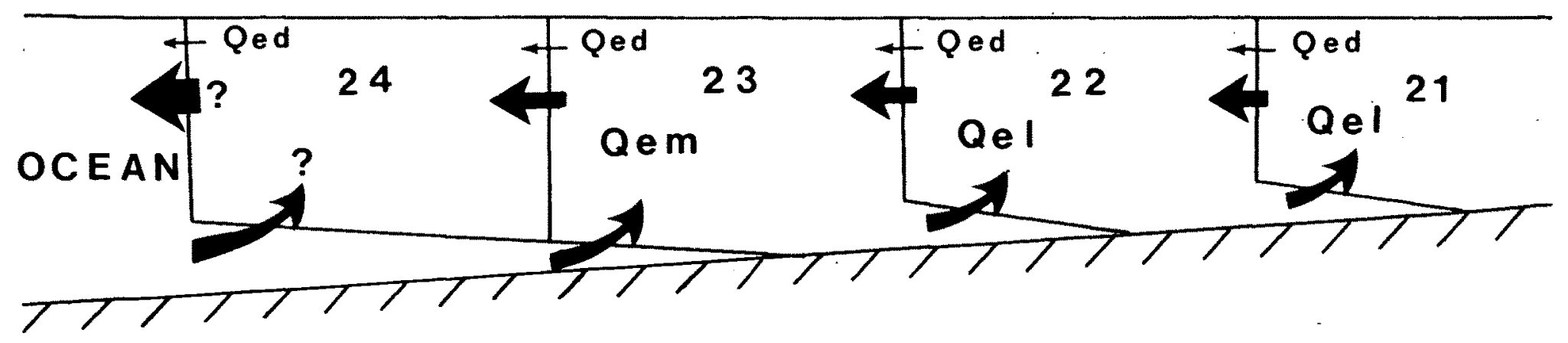

Qed = Débit d'eau douce Qem = Débit d'eau de mer Qel = Débit des échanges longitudinaux

Fig. 3. Découpage utilisé pour les calculs dans la zone aval de l'estuaire et représentation des débits résiduels.

Fig. 3. Divisions used for calculations in the lower part of the estuary and representation of residual flows.

n'est pas pris en considération. Il s'agit en effet, d'un large bassin à l'abri de digues. Ses caractéristiques sont très différentes de celles de l'estuaire proprement dit et il n'a pas pu être étudié en détail.

Ces 23 tronçons sont considérés comme homogènes du point de vue de la salinité. La longueur des tronçons $(1000 \mathrm{~m})$ a été choisie de façon à ce que la différence de salinité entre les tronçons successifs soit du même ordre de grandeur que la différence de salinité maximum mesurée par Mahif (1987) entre le fond et la surface $(1 \mathrm{~g} / 1)$. Les limites de chaque tronçon suivent les courbes d'isosalinité observées. Elles sont pratiquement verticales sur la plus grande partie de la section puis s'incurvent vers l'amont au voisinage du fond. Une partie de l'eau de chaque tronçon s'engage ainsi vers l'amont sous la tronçon suivant pour former une sorte de micro coin salé (Fig. 3). La forme de cette courbure étant à peu près identique à l'amont et à l'aval de chaque portion, la salinité moyenne de chaque tronçon peut être calculée sans tenir compte des légères différences existant entre le fond et la surface.

Les apports d'eau salée se font au travers de l'interface aval de chaque tronçon notamment au niveau du micro coin salé (le voisinage du fond correspond à une zone d'augmentation de la turbulence). Les sorties d'eau mélangée se font essentiellement au travers de la partie verticale de l'interface aval de chaque tronçon. Dans le modèle morphologique, l'estuaire est représenté invariablement à mimarée. Les entrées et sorties d'eau décrites ci-dessus correspondent ainsi aux débits résiduels moyens.

Le modèle de fonctionnement choisi est donc un modèle filaire de mélange des eaux permettant de calculer l'évolution de la salinité moyenne à mi- marée dans les 23 tronçons de l'estuaire connaissant les apports d'eau douce et l'importance des débits résiduels.

\section{Modélisation des débits résiduels dans l'estuaire du Bou Regreg}

En raison de l'existence d'un micro coin salé à l'interface de chaque tronçon, on peut considérer chaque fois cette zone comme un modèle réduit d'estuaire stratifié avec entraînement. Chaque tronçon joue ainsi, pour le reste de l'estuaire sité en amont, le rôle que joue la mer dans les modèles généraux d'estuaires stratifiés.

Pour un estuaire stratifié avec entraînement (Fig. 4), il est possible de calculer les débits résiduels grâce à la méthode décrite par Bowden (1967) en connaissant seulement la valeur des apports d'eau douce et la salinité moyenne de l'eau sortant de l'estuaire en période de strict équilibre. Pour être applicable au Bou Regreg, cette méthode doit être légèrement modifiée pour tenir compte de la salinité de l'eau douce reçue par l'estuaire.

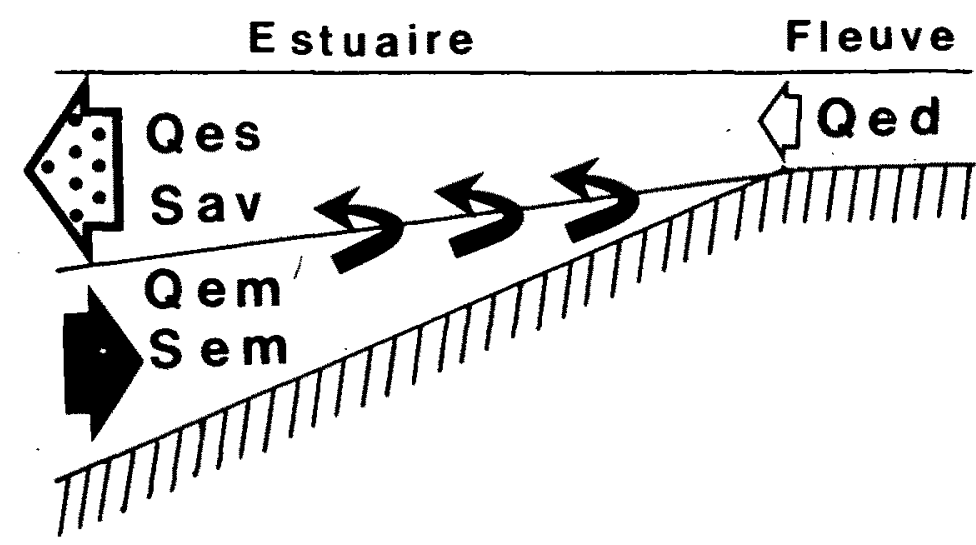

Fig. 4. Représentation schématique du fonctionnement d'un estuaire stratifié.

Fig. 4. Schematic representation of a stratified estuary. 
Celle-ci, en effet, ne peut pas être négligée puisqu'elle présente une salinité six fois plus élevée que la moyenne des eaux douces européennes calculée par Meybeck (1979).

Il s'agit d'écrire les équations de bilan de la masse d'eau (I) et de sel (II) en amont de chaque tronçon. (I) $\mathrm{Qs}=\mathrm{Qe}+\mathrm{Qed}$

avec : Qs = Débit résiduel de l'eau mélangée sortant du tronçon

$\mathrm{Qe}=$ débit résiduel d'entrée de l'eau venant du tronçon aval

Qed = Débit d'entrée de l'eau douce

(II) $\quad$ Qs $\times$ Ss $=$ Qe $\times$ Se + Qed $\times$ Sed

avec : Ss = Salinité de l'eau sortant de l'estuaire

$\mathrm{Se}=$ Salinité de l'eau venant du tronçon aval

Sed $=$ Salinité de l'eau douce $=0,6 \mathrm{~g} / 1$

d'où : Qe = Qed (Ss-Sed) / (Se-Ss).

La résolution de cette dernière équation se fait tronçon par tronçon de l'aval vers l'amont.

Cette méthode est, dans la pratique, limitée par les difficultés de mesure des débits d'eau douce (une partie des apports peuvent être diffus), par les variation de ces débits et par la durée du transfert des masses d'eau entre le fleuve proprement dit et l'embouchure. Les variations de la salinité d'un tronçon à l'autre sont également difficiles à apprécier ; elles sont très faibles vers l'aval et peuvent être perturbées par des phénomènes passagers tels que le vent... La situation d'équilibre parfait, nécessaire pour que le modèle ci-dessus fournisse des résultats précis, n'est en fait jamais atteinte dans un estuaire réel.

Il est possible de contourner une grande partie de zes difficultés en ne prenant pas pour référence une période de stabilité relative mais au contraire une période de retour vers l'équilibre après une perturbation importante comme un lâcher de barrage. En əffet, le phénomène mesurable de remontée de l'eau le mer dans l'estuaire après une forte baisse de la :alinité suivie d'apports d'eau douce réduits et à peu orès stables, correspond à une combinaison de deux ypes d'apports que l'on peut évaluer séparément.

On peut tout d'abord calculer, grâce au modèle :i-dessus dérivé de celui de Bowden, les débits noyens d'eau salée entrant dans l'estuaire pour équiibrer les arrivées d'eau douce. Pour cela, on utilise dans les calculs les salinités moyennes observées au cours de la période suivant le lâcher.

Parallèlement, il existe des entrées supplémentaires d'eau salée responsables de l'élévation de la salinité indépendamment des arrivées d'eau douce. $\mathrm{Ce}$ dernier phénomène correspond à ce qui se passe dans un estuaire ou une lagune lorsqu'ils ne reçoivent aucun apport d'eau douce pendant un certain temps (Proudman 1953).

Dans ce cas, le bilan des masses de sel s'écrit de la façon suivante :

$(\mathrm{Sa} 1-\mathrm{Sa} 2) \mathrm{Va}=\mathrm{Vem} \times \mathrm{Sem}-\operatorname{Vav} \times \mathrm{Sav}$ Avec : Sa1 = Salinité moyenne en amont de l'embouchure au temps $t 1$.

$\mathrm{Sa} 2=$ Salinité moyenne en amont de l'embouchure $\mathrm{t} 2$.

$\mathrm{Va}=$ Volume de l'estuaire à mi-marée en amont de l'embouchure (de la mer à la limite de l'intrusion saline).

Vem $=$ Volume résiduel d'eau de mer entrant dans l'estuaire.

Vav $=$ Volume résiduel d'eau mélangée sortant de l'estuaire.

Sem $=$ Salinité de l'eau de mer.

Sav = Salinité moyenne de l'eau sortant de l'estuaire pendant la période considérée. L'augmentation de la salinité étant supposée régulière : Sav $=(\mathrm{S}$ au temps $\mathrm{t} 1+\mathrm{S}$ au temps $\mathrm{t} 2$ ) $/ 2$.

En l'absence de débit fluvial Vem = Vav, d'où $(\mathrm{S} 1-\mathrm{S} 2) \mathrm{Va}=$ Vem $($ Sem - Sav). Si $\mathrm{t}=$ durée de la période considérée, le débit d'eau de mer responsable de la ressalure peut s'écrire : Quem $=$ Vem $/ \mathrm{t}=(\mathrm{S} 1$ - S2) $\mathrm{Va} /$ Sem $($ Sem - Sav) $\mathrm{t}$.

Comme avec le premier modèle, on calcule ces débits résiduels entre les tronçons successifs en prenant chaque tronçon comme s'il représentait la mer pour le reste de l'estuaire situé en amont.

Lorsque l'on a calculé, grâce aux deux modèles ci-dessus, les débits résiduels équilibrant les arrivées d'eau douce et les débits résiduels responsables de la remontée de la salinité, on les additionne pour obtenir les débits résiduels effectifs correspondant à la moyenne des marées enregistrées au cours de la période considérée. 


\section{Matériel et méthodes}

\subsection{Echantillonnage et mesures}

Les prélèvements d'eau ont été effectués le même jour aux étales de marée haute et de marée basse en mettant à profit les retards de propagation de l'onde de marée à l'intérieur de l'estuaire.

L'eau a été prélevée à l'aide de bouteilles en polyéthylène au voisinage du bord, à $10 \mathrm{~cm}$ audessous de la surface. Les stations de prélèvement ont été choisies et testées de façon à obtenir des échantillons aussi représentatifs que possible. Il a été tenu compte en particulier de la position des rejets d'égoûts par rapport aux points de prélèvement. L'hétérogénéité verticale a été étudiée par Mahif (1987) qui a trouvé des différences minimes dans la répartition de la salinité (au maximum $1 \mathrm{~g} / \mathrm{l}$ de plus près du fond). Les échantillons prélevés en surface ont été toutefois considérés comme suffisamment représentatifs de la tranche d'eau la plus importante qui glisse progressivement vers l'aval.

La salinité totale a été utilisée comme traceur naturel. Elle a été calculée à partir de mesures de conductivité électrique effectuées au laboratoire, le jour même du prélèvement, à l'aide d'un conductimètre TACUSSEL CD. $6 \mathrm{~N}$. Elle représente la masse totale de sels exprimée en grammes par litre de solution.

\subsection{Fréquence des prélèvements}

La période choisie pour étalonner le modèle des débits résiduels est consécutive à un important lâcher de barrage qui a eu lieu en février 1987 (10 jours de lâcher avec un débit moyen de $\left.150 \mathrm{~m}^{3} . \mathrm{s}^{-1}\right)$. Pour étudier cette période, 3 séries de mesures de la salinité à marée haute et marée basse ont été effectuées en 8 points de l'estuaire (Dahbi 1988).

- Série 1 : Prélèvements au cours du lâcher : l'eau de surface est partout de l'eau douce. L'estuaire présente une stratification temporaire en aval alors que l'eau salée est totalement chassée de la partie amont.

- Série 2 : Prélèvements 9 jours après l'arrêt du lâcher : la salinité en surface s'est élevée très rapidement dès que l'estuaire est retourné à une situation d'homogénéité verticale, puis elle a augmenté beaucoup plus lentement.
Série 3 : Prélèvements 49 jours après l'arrêt du lâcher. La salinité est à nouveau voisine de ce qu'elle était avant le lâcher.

L'importance de l'écart entre les salinités observées entre le $9^{\mathrm{e}}$ et le $49^{\mathrm{e}}$ jour après l'arrêt du lâcher (Fig. 5) permet de limiter les effets des erreurs d'échantillonnage et de mesure au moins dans la partie moyenne de l'estuaire.

\subsection{Paramètres utilisés dans les modèles}

Tous les calculs ont été effectués à partir des moyennes de salinité entre marée haute et marée basse pour chacune des 8 stations. Faute de mieux, ces moyennes ont été assimilées à la salinité à mimarée. La salinité moyenne de chaque portion de $1 \mathrm{~km}$ de l'estuaire a ensuite été calculée par interpolation (Tableau 1, Fig. 5).

La salinité de l'eau de mer entrant dans l'estuaire au cours de la période a été estimée à $30.7 \mathrm{~g} . \mathrm{l}^{-1} \mathrm{ce}$ qui correspond à la moyenne entre les salinités à marée haute à l'embouchure, observées 9 jours et 49 jours après l'arrêt du lâcher.

Les apports d'eau douce ont été évalués à partir d'études antérieures, comme pour l'égout du quartier Douar Doum (Cheddadi 1987) ou les fuites du barrage, et d'observations récentes associées à des calculs de contribution relative comme pour l'Oued Akkrech. Les pluies ayant été très limitées au cours de la période suivant le lâcher, les apports naturels sont demeurés faibles et réguliers. Les apports des égouts ont été ainsi estimés à $100 \mathrm{l} . \mathrm{s}^{-1}$, ceux de l'Oued Akkrech à $601 . \mathrm{s}^{-1}$ et les fuites du barrage à $301 . \mathrm{s}^{-1}$.

Grâce à l'étude du déplacement des masses d'eau en fonction de la marée effectuée par Dahbi (1988) il a pu être affecté à chaque tronçon de l'estuaire la part d'eau douce qui lui est revenue en moyenne par unité de temps au cours de la période considé rée (Tableau 1, Fig. 6).

\section{Validation du modèle des débits résiduel par un modèle de mélange}

Grâce aux deux modèles décrits au chapitre 4 le débits résiduels globaux s'établissant entre les tron çons successifs ont pu être calculés (Tableau 1, Fig 3 et 6$)$.

La connaissance des débits résiduels est un préa lable qui permet d'élaborer un modèle global de 

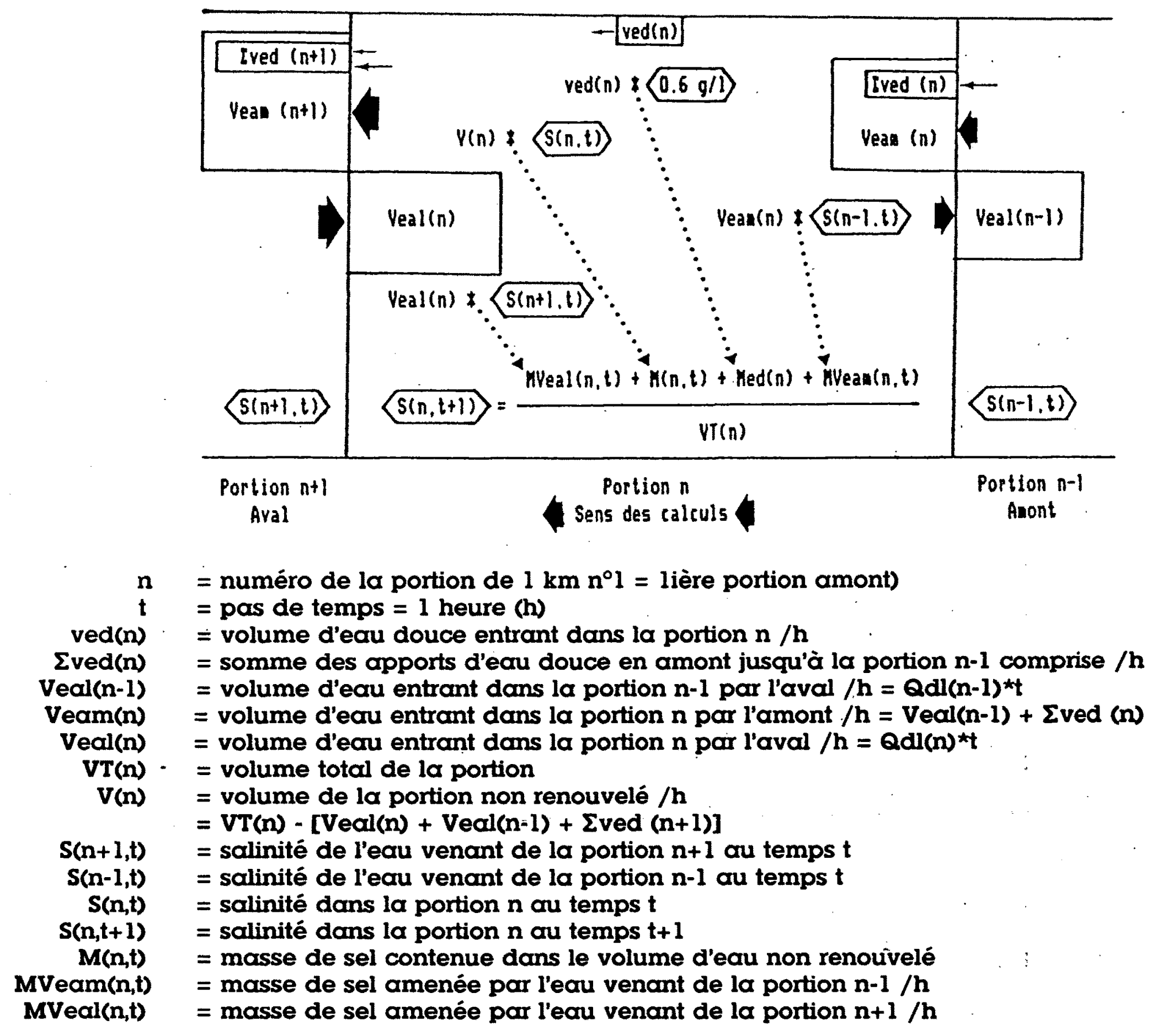

Fig. 5. Opérations effectuées pour calculer l'évolution de la salinité dans un estuaire verticalement homogène.

Fig. 5. Operations needed to calculate the salinity changes in a vertically homogeneous estuary.

mélange (Fig. 5). Chaque tronçon de $1 \mathrm{~km}$ échange en permanence de l'eau avec le tronçon amont et le tronçon aval. Il reçoit en plus, éventuellement, un apport d'eau douce latéral. A ceci vieǹt s'ajouter un glissement vers l'aval correspondant à la somme des apports d'eau douce existant en amont. Ce modèle permet d'effectuer une simulation de la remontée de la salinité dans l'estuaire entre le $9^{e}$ et le $49^{\mathrm{e}}$ jour après le lâcher.
Les calculs de salinité ont été effectués kilomètre par kilomètre et heure par heure en utilisant l'équation de bilan de sel suivante :

Connaissant la masse de sel au temps $t$ dans chaque portion, la masse de sel présente dans la portion $\mathrm{n}$ au temps $\mathrm{t}$ s'écrit :

$$
\begin{gathered}
M(n, t+1)=Q d l(n) \times 3600 \times S(n+1, t)+V(n) \\
\times S(n, t)+Q e d(n) \times 3600 \times 0.6+Q d l(n-1, t) \\
\times 3600 \times S(n-1, t) \\
\text { d'où } S(n, t+1)=M(n, t+1) / V T(n)
\end{gathered}
$$


Les résultats obtenus paraissent satisfaisants. L'écart entre les salinités observées le $49^{e}$ jour après le lâcher et les salinités calculées est au maximum de $1.13 \mathrm{~g} . \mathrm{l}^{-1}(4 \%)$ et l'écart absolu moyen est de 0,44 g. $1^{-1}(2 \%)$ (Tableau 1, Fig. 7 et 8 ).

La concordance entre les salinités calculées et les salinités mesurées confirme la validité des hypothèses de départ, des méthodes de calcul des débits résiduels et des estimations du débit des arrivées d'eau douce.

\section{Discussion}

Le modèle proposé est un modèle de mélange ou de dilution qui ne prend qu'indirectement en compte les phénomènes hydrodynamiques proprement dits. Il est nécessaire de préciser ses limites de validité qui sont fonction de son architecture propre et de la nature des données de départ.

Le modèle ci-dessus a été élaboré à partir de données recueillies à 40 jours d'intervalle. Au cours de cette période, l'amplitude moyenne de la marée $(1.9 \mathrm{~m}) \mathrm{a}$ été légèrement inférieure à la moyenne annuelle $(2 \mathrm{~m})$.

Il est possible cependant d'utiliser le modèle pour d'autres périodes de durée inférieures ou supérieures à 40 jours, de préférence par tranches de 15 jours, si l'amplitude moyenne de la marée reste voisine de $1.9 \mathrm{~m}$. Il faut également que l'amplitude de la marée soit voisine de $2 \mathrm{~m}$ au début et à la fin

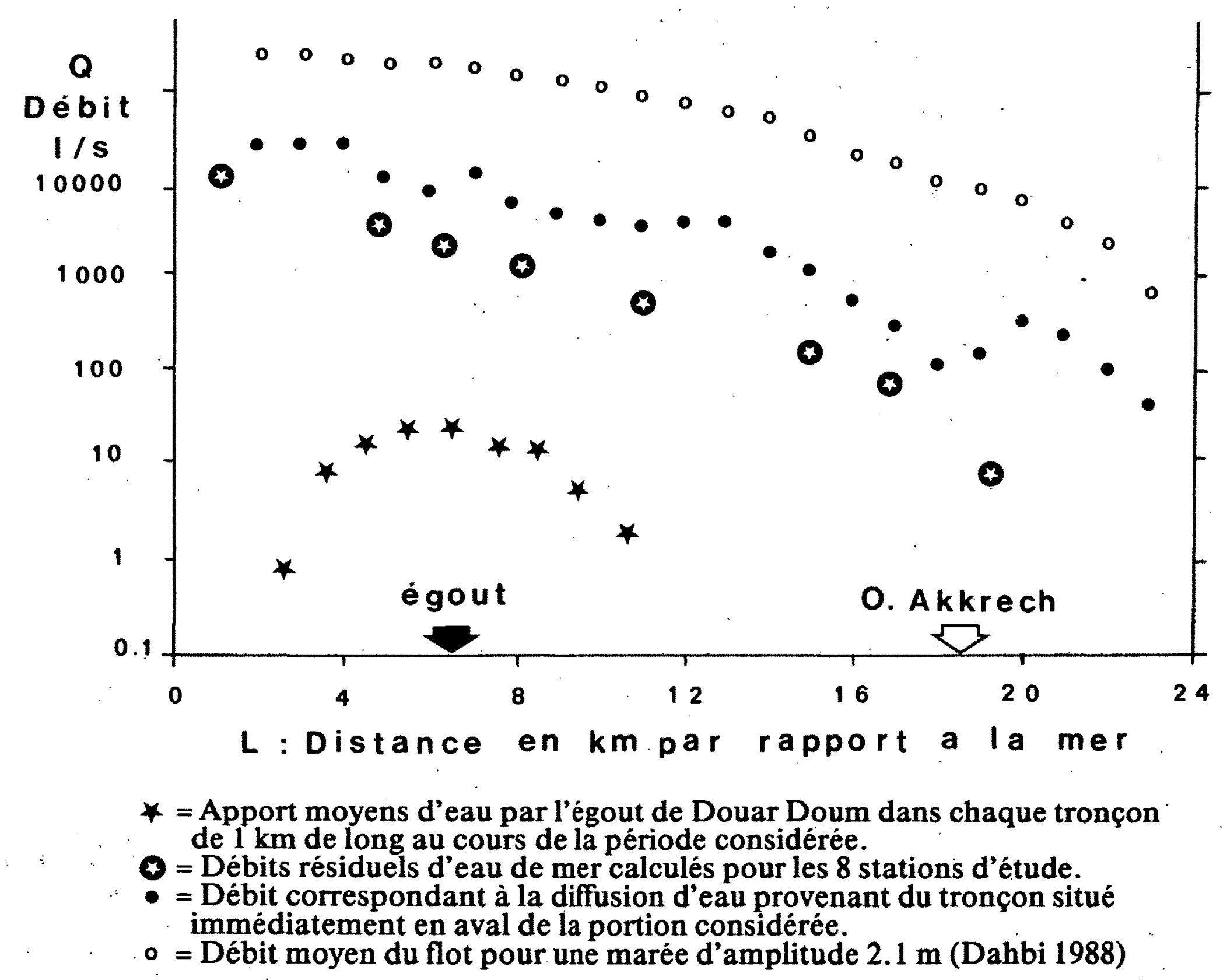

Fig. 6. Débits d'eau impliqués dans les variations de la salinité à l'intérieur de l'estuaire du Bou Regreg en Mars Avril 1986.

Fig. 6. Water discharges involved in the salinity variations along the Bou Regreg estuary during Marc and April 1986. 
de chaque période considérée. En effet, en plus des problèmes liés aux variations des courants de marée en fonction du marnage, la présence d'un seuil au niveau des îles perturbe le fonctionnement de l'estuaire. Il a pu être effectivement constaté que la zone située en amont des îles fonctionne comme un réservoir qui se remplit plus facilement qu'il ne se vide. Ceci accentue les différences entre les périodes de mortes eaux et de vives eaux et même entre les périodes d'augmentation et de diminution du coefficient de marée.

La figure 9 montre 2 exemples de simulation de la variation de la salinité comparée aux variations observées. Les résultats sont concordants aussi bien pour une période d'augmentation de la salinité comparable à celle qui a servi de base pour l'élaboration du modèle que pour une période de diminution de la salinité consécutive à des précipitations abondantes.

Il apparaît donc que si les apports d'eau douce sont correctement évalués et que les recommandations énoncées ci-dessus sont prises en compte, le modèle de mélange peut servir de modèle de prédétermination quelle que soit la situation envisagée. Il est en particulier possible, moyennant certaines modifications, d'utiliser aussi ce modèle pour simuler la dispersion d'une substance dissoute introduite en n'importe quel point de l'estuaire.

La connaissance du temps de transit de l'eau entre l'amont et l'embouchure (flushing time) est d'un très

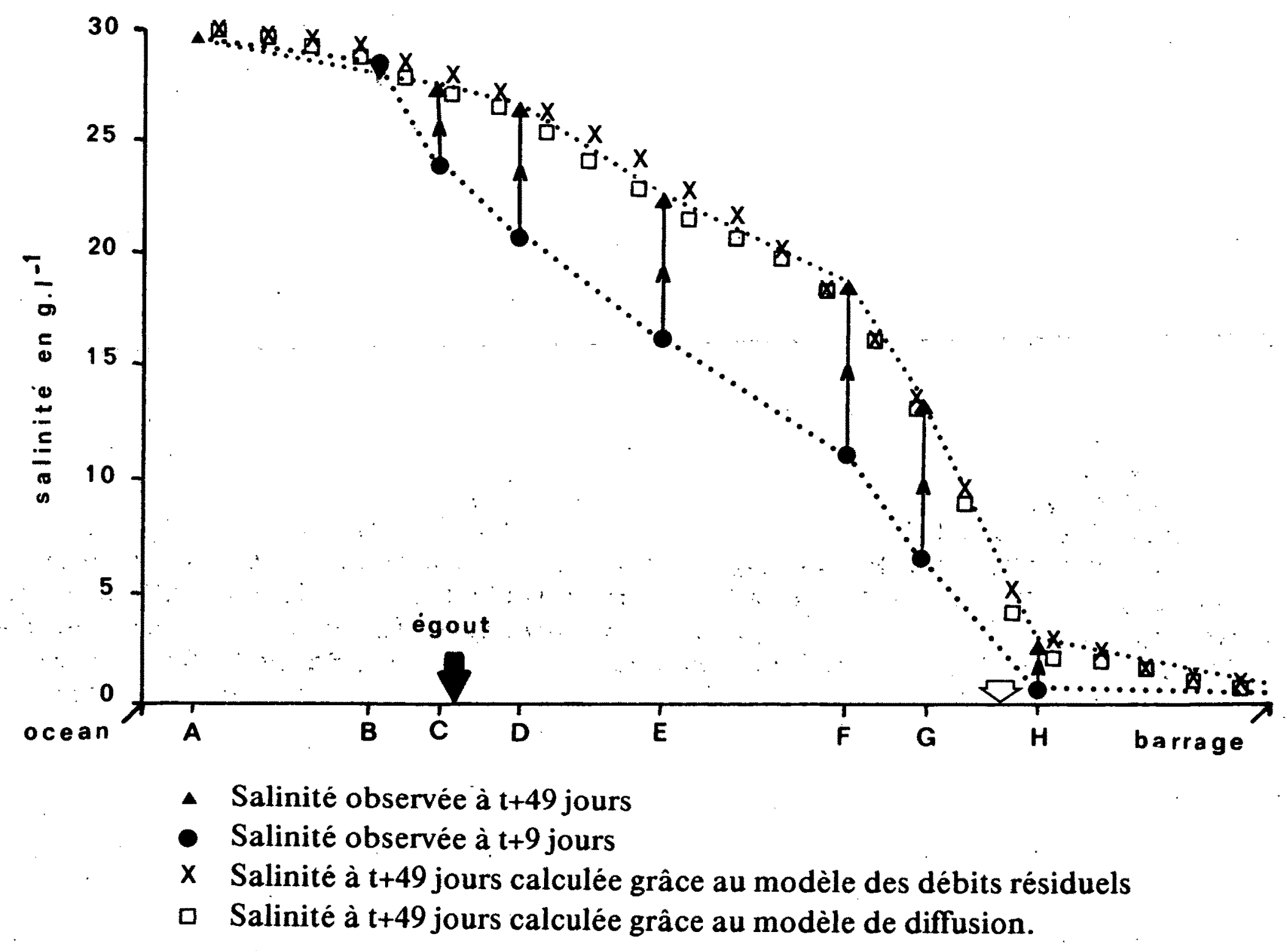

Fig. 7. Evolution de la salinité le long de l'estuaire du Bou Regreg au cours d'une période de 40 jours suivant un lâcher du barrage situé en aval.

Fig. 7 Salinity changes along the Bou Regreg estuary over 40 days following a release of fresh water from the upstream reservoir. 
grand intérêt pratique mais il est très difficile de le calculer avec une grande précision (Pilson 1985).

La méthode des salinités, proposée par Bowden (1967), permet d'abord de calculer la quantité d'eau douce (Ved) se trouvant dans l'estuaire à un moment donné. Si le débit fluvial est alors Qed, le temps de résidence moyen de l'eau douce dans l'estuaire est égal à Ved/Qed. Malgré ses nombreuses imperfections, cette méthode est toujours employée et fournit des ordres de grandeur vraisemblables (Castaing 1981).

. Pour l'estuaire du Bou Regreg, le calcul du temps de résidence moyen n'a de sens qu'en aval du confluent avec l'Oued Akkrech, la partie amont n'étant alimentée que par les fuites du barrage.

Si l'on considère les salinités observées en période de relative stabilité, pour des débits d'eau douce estimés à $1901 . \mathrm{s}^{-1}$ le temps de résidence moyen de l'eau douce en aval de l'Oued Akkrech calculé par la méthode de Bowden est de 84 jours.

Un modèle des débits résiduels d'eau de mer « pure " de l'embouchure à l'Oued Akkrech a également été élaboré. Il s'agit d'un modèle d'estuaire stratifié avec entraînement dont la structure est très voisine de celle du modèle précédent mais qui suppose la présence d'une couche d'eau de mer sous la couche d'eau mélangée. Ce modèle ne prétend pas représenter la réalité physique de l'estuaire mais on peut l'utiliser pour simuler l'évolution de la salinité au cours de la période étudiée (Fig. 8). Les résultats obtenus sont très voisins der ceux du modèle de mélange (écart moyen par rapport à la salinité mesurée $=7,4 \%$ )... Ce modèle permet surtout de calculer un temps de résidence moyen des eaux dans chacune des 23 portions de l'estuaire. On obtient ainsi un temps de résidence total de 86 jours en aval de l'Oued Akkrech. La concordance entre les deux résultats obtenus signifie entre autres que les débits d'eau douce ont été correctement estimés.

Ces deux modèles peuvent trouver des applications dans les études d'impact des aménagements qui sont en cours de réalisation le long de l'estuaire du Bou Regreg. Il est possible par exemple, de démontrer que le déplacement de seulement 1 kilomètre vers l'aval de l'émissaire des égouts de Douar Doum ferait passer de 9.9 à 6.9 jours le temps de résidence moyen dans l'estuaire des eaux apportées par ces égouts ce qui correspondrait à une diminution du temps de séjour de $30 \%$.

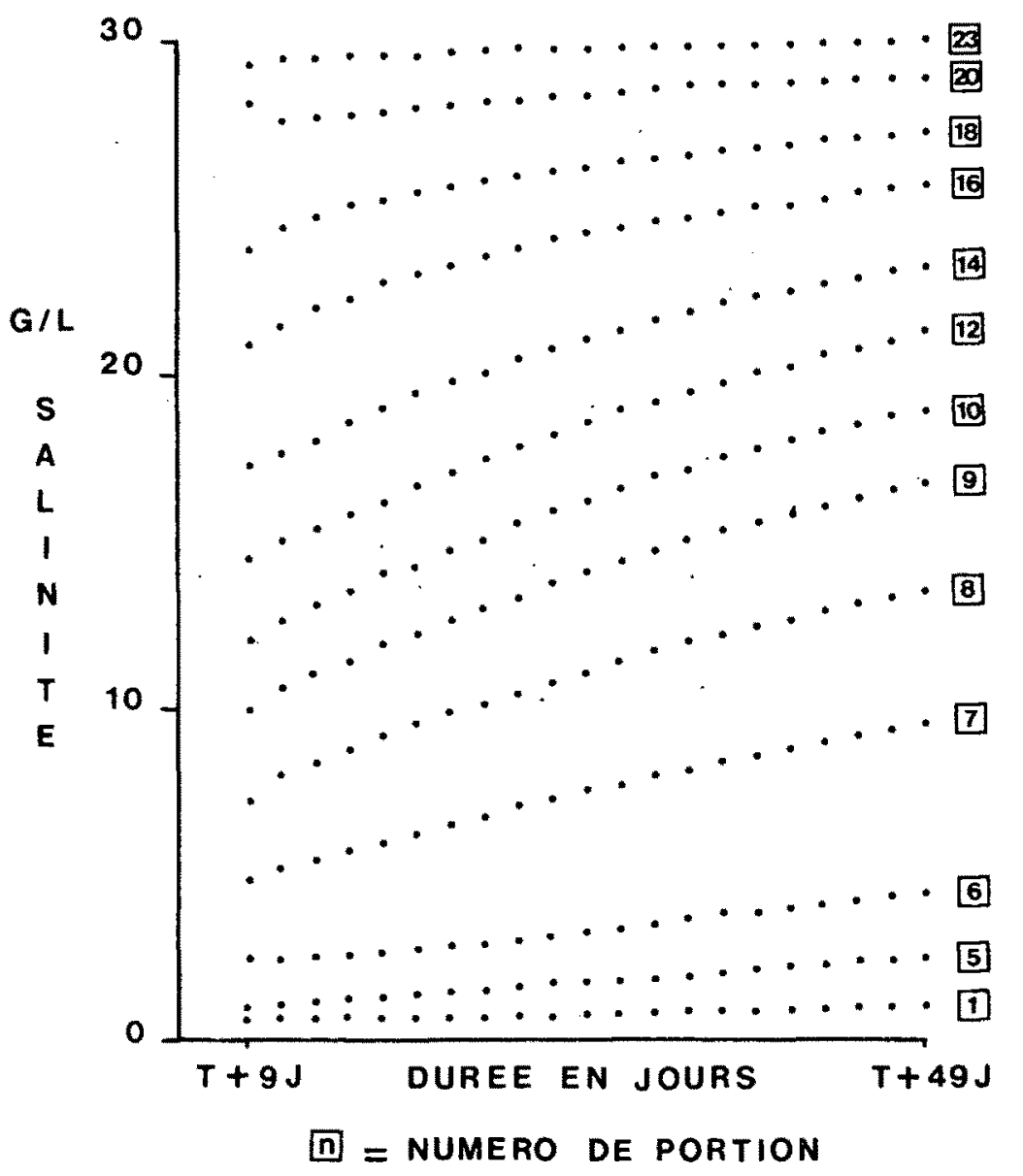

Fig. 8. Evolution en fonction du temps de la salinité calculée par le modèle de mélange au cours d'une période de 40 jours suivant un lâcher du barrage.

Fig. 8. Salinity changes over time calculated by the mixing model over 40 days following a release from a reservoir.

\section{Conclusion}

La mise à profit de la période suivant une crue ou un lâcher de barrage peut permettre l'élaboration relativement rapide d'un modèle de mélange des eaux dans des estuaires comme celui du Bou Regreg. Les estuaires de ce type, qui, au cours de l'année, demeurent la plupart du temps verticalement homogènes sont de plus en plus nombreux dans des pays tels que le Maroc. En effet, dans toutes les zones où sévit une sécheresse chronique, la pression humaine sur les ressources en eau est si forte que de moins en moins d'eau douce parvient à la mer. Corrélativement, l'eau salée remonte de plus en plus vers l'amont des estuaires et y séjourne de plus en plus longtemps.

Tout se passe comme si chacun de ces estuaires subissait une translation progressive vers une zone de plus grande aridité. Cette évolution peut être accélérée lors de la mise en service d'un barrage. Il est intéressant d'étudier ces changements qui 

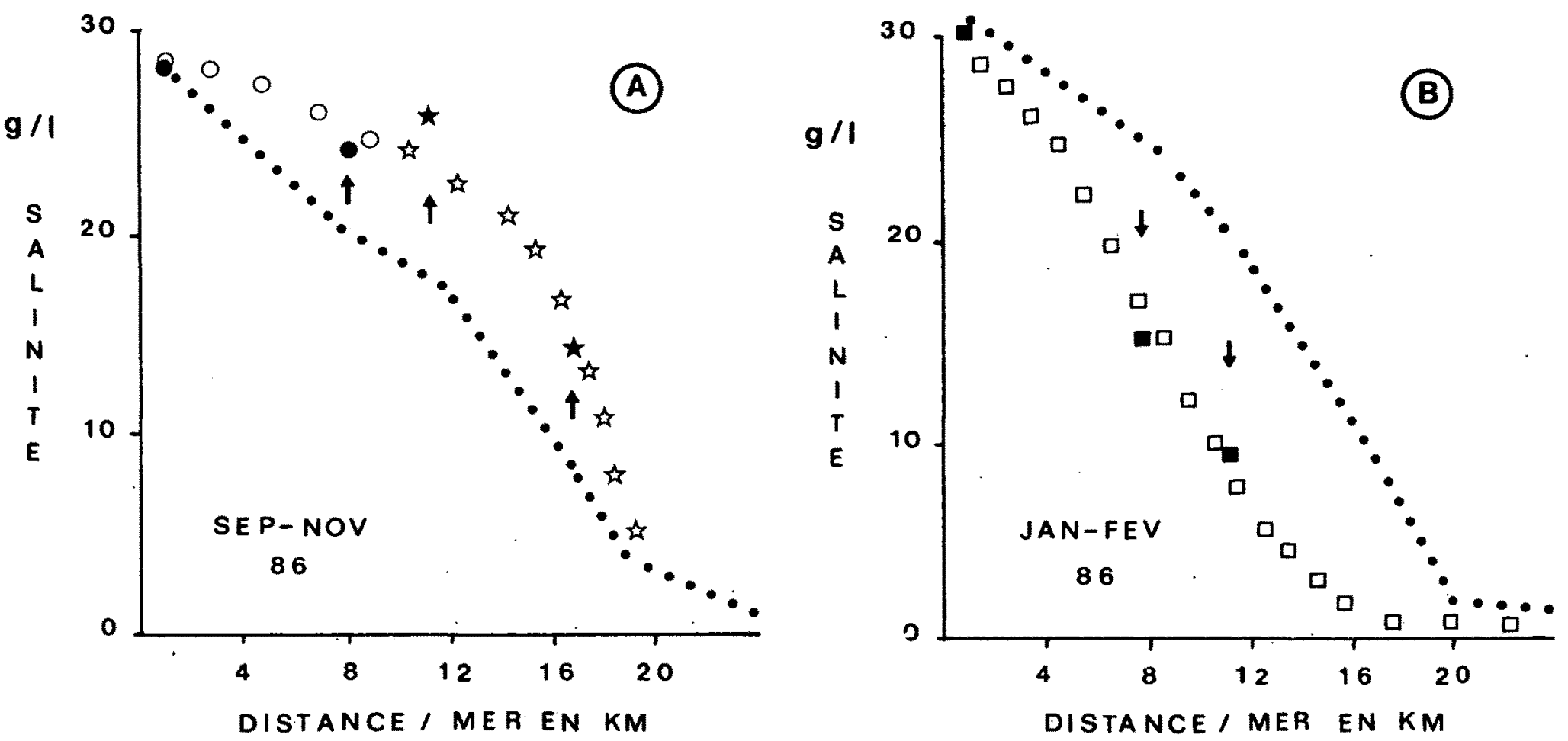

Fig. 9. Salinité à mi-marée calculée par le modèle de mélange et salinité observée au cours de deux périodes de l'année 1986. Le trait en pointillés représente la salinité au début de la période considérée.

A la fin de chaque période, la salinité calculée grâce au modèlè est représentée par les figurés clairs alors que la salinité mesurée est représentée par les figurés noirs.

$\mathbf{A}=$ Période de remontée de l'eau de mer dans l'estuaire. Les cercles représentent la salinité au bout de 39 jours. Les étoiles représentent la salinité au bout de 49 jours. $\mathrm{B}=$ Période de 28 jours incluant de fortes précipitations. Le débit de l'oued Akkrech est estimé à $1225 \mathrm{~V} / \mathrm{s}$.

Fig. 9. Salinity at midtide calculated with mixing model and salinity observed during two periods for the year 1986.

The dotted line indicates the salinity at the begininng of each period.

At the end of each period, the salinity calculated by the model is indicated by clear symbols and the measured salinity is indicated by black symbols. $A=$ Period of sea water entering the estuary. The circles indicate the salinity after 39 days. The stars indicate the salinity after 49 days. B $=28$ day - period including heavy precipitation. The Oued Akkrech discharge has been assessed at $1225 \mathrm{l} / \mathrm{s}$.

influencent fortement la répartition de la faune estuarienne et qui peuvent par ailleurs avoir des conséquences économiques et sanitaires non négligeables.

\section{Remerciements}

L'auteur remercie M. Mahif, M. Cheggour et N. Dahbi qui ont effectué le travail de terrain et de laboratoire sans lequel l'élaboration des modèles présentés ci-dessus aurait été impossible.

\section{Travaux cités}

Arons A.B. \& Stommel H. 1951. - A mixing length theory of tidal flushing. Trans. Am. Geophys. Un., $32: 419-421$.

Bowden K.F. 1967. - Circulation and diffusion. In Estuaries, G.H. Lauff éd. Washington. 15-36.

Bradley P.M., Kjerfve B. \& Morris J.T. 1980. - Rediversion salinity change in the Cooper River, South Carolina : Ecological implications. Estuaries, 13 (4) : 373-379.

Brown W.S. \& Arellano E. 1980. The application of a segmented tidal mixing model to the Great Bay Estuary, N.H. Estuaries, 4 (3) : 248-257.
Castaing P. 1981. - Le transfert à l'océan des suspensions estuariennes - cas de la Gironde. Thèse Doct. ès Sc. Univ. Bordeaux : $530 \mathrm{p}$.

Cheggour M. 1988. - Contribution à l'étude de l'estuaire du Bou Regreg (côté atlantique marocaine). Conditions écologiques globales, étude de la contamination métallique. Thèse de doctorat $3^{e}$ cycle, Ecole Normale Supérieure Takadoum, Rabat, 337 p.

Cheggour M., Texier H., Moguedet G. \& Elkaïm B. 1990. Metal exchanges in the fauna-sédiment system. The case of Nereis diversicolor and Scrobicularia plana in the Bou Regreg estuary (Morocco). Hydrobiologia, 207 : 209-219.

Dahbi N. 1988. - Contribution à l'étude de l'estuaire du Bou Regreg (côte atlantique marocaine) - Hydrologie - Hydrodynamique - Pollution métallique. Thèse de doctorat $3^{\mathrm{e}}$ cycle, Ecole Normale Supérieure Takadoum, Rabat. 255 p.

Dyer K.R. \& Taylor P.A. 1973. - A segmented prism model of tidal mixing. Estuarine and Coastal Marine Science 1 : 411-418.

Elkaïm B. 1972. - Contribution à l'étude d'un estuaire atlantique marocain : l'estuaire du Bou Regreg. Bull. Soc. Sci. nat. Phys. Maroc. 52 : 131-339.

Elliot A.J. 1976. - A mixed-dimension kinematic estuarine model. Cheseapeake Science, 17 (3) : 135-140. 
Fisher H.B. 1972. - Mass transport mechanisms in partially stratified estuaries. J. Fluid Mech. 53 (4) : 671-687.

Geyer W.R. \& Signell R.P. 1992. - A reassessment of the role of tidal dispersion in estuaries and bays. Estuaries, 15 (2) : 97-108.

Gillet P. 1987. - Contribution à l'étude écologique des annélides polychètes de l'estuaire du Bou Regreg (Maroc). Thèse de doctorat, Université Aix Marseille III : $187 \mathrm{p}$.

Hansen D.V. \& Rattray M. 1966. - New dimensions in estuary classification. Limnol. Oceanogr. 11 (3) : 319-326.

Ketchum B.H. 1951. - The exchange of fresh and salt waters in tidal estuaries. $J$. Mar. res. $10: 18-38$.

Mayif M.O.A. 1987. - Un modèle estuarien limite : le Bou Regreg (Maroc). Contribution à l'étude hydrodynamique, hydrologique, géochimique et minéralogique. Thèse de doctorat $3^{e}$ cycle, Ecole Normale Supérieure Souissi, Rabat : $136 \mathrm{p}$.

Meybeck M. 1979. - Concentration des eaux fluviales en éléments majeurs et apports en solution aux océans. Rev. Geol. Dyn. Geog. Phy. 21 (3) : 215-246.

Meyer-Branski B. 1981. - Transfert dispersif en estuaire par simulation numérique. Thèse de docteur ingénieur I.N.P. Toulouse : $96 \mathrm{p}$.

Pilson M.E.Q. 1985. - On the residence time of water in Narraganset Bay. Estuaries 1 (8) : 2-14.
Preddy W.S. 1954. - The mixing and movement of water in the estuary of the Thames. J. Mar. Biol. Assoc. U.K. 33 : 645-662.

Pritchard D.W. 1955. - Estuarine circulation patterns. Proc. Am. Civ. Engin. 81 : 1-11.

Pritchard D.W. 1967. - Observations of circulation in coastal plain estuaries. In Estuaries, G.H. Lauff ed., Washington : 15-36.

Proudman J. 1953. - Dynamical oceanography. Wiley ed., New York : 409 p.

Simpson J.H., Brown J., Matthews J. \& Allen G. 1990. - Tidal straining, density currents, and stirring in the control of estuarine stratification. Estuaries 13 (2) : 125-132.

Salomon J.C., Breton M. \& Pommepuy M. 1991. Intérêt d'un modèle de transports dissous pour les rejets urbains en zone estuarienne. In La mer et les rejets urbains, Actes de colloques $n^{\circ} 11$. IFREMER. Brest : 205-220.

Texier H., Mayif M.O.A., Nasseh A. \& Teffal M. 1986. - Contribution à l'étude hydrologique d'un modèle estuarien limite le Bou Regreg (Maroc). In Fac. sci. Marrakech, $1^{\text {er }}$ colloque franco-marocain sur l'eau, $1: 56$.

Ulanowicz R.E. 1976. - Modelling the Chesapeake Bay and tributaries : synopsis. Chesapeake Science. 17 (2) : 114-122. 